\title{
Erratum to: Prevalence of chronic kidney disease in the Japanese general population
}

\author{
Enyu Imai - Masaru Horio · Tsuyoshi Watanabe $\cdot$ Kunitoshi Iseki · Kunihiro Yamagata $\cdot$ Shigeko Hara \\ Nobuyuki Ura $\cdot$ Yutaka Kiyohara · Toshiki Moriyama • Yasuhiro Ando · Shoichi Fujimoto • \\ Tsuneo Konta $\cdot$ Hitoshi Yokoyama $\cdot$ Hirofumi Makino $\cdot$ Akira Hishida $\cdot$ Seiichi Matsuo
}

Published online: 22 October 2009

(C) Japanese Society of Nephrology 2009

\section{Erratum to: Clin Exp Nephrol \\ DOI 10.1007/s10157-009-0199-x}

In Table 3, in the column headed "Proteinuria (+)", the "Estimated number of Japanese adults in 2005" in the

The online version of the original article can be found under doi:10.1007/s10157-009-0199-x.

\section{E. Imai ( $\square)$}

Department of Nephrology,

Osaka University Graduate School of Medicine,

Suita, Osaka 565-0871, Japan

e-mail: ADS12069@nifty.com

\section{Horio}

Department of Functional Diagnostic Science,

Osaka University Graduate School of Medicine,

Osaka, Japan

\section{T. Watanabe}

Third Department of Medicine,

Fukushima Medical University, Fukushima, Japan

K. Iseki

Dialysis Unit, University Hospital of the Ryukyus,

Nishihara, Okinawa, Japan

\section{K. Yamagata}

Department of Nephrology, Institute of Clinical Medicine, Graduate School of Comprehensive Human Sciences,

University of Tsukuba, Ibaraki, Japan

\section{S. Hara}

Health Medical Center, Toranomon Hospital,

Tokyo, Japan

N. Ura

Department of General Medicine,

Teine Keijinkai Hospital, Sapporo, Hokkaido, Japan
30-59 age-group should be 823881 , not 8238881 . The corrected table is shown here.

\author{
Y. Kiyohara \\ Department of Environmental Medicine, Graduate School \\ of Medical Sciences, Kyushu University, Fukuoka, Japan \\ T. Moriyama \\ Healthcare Center, Osaka University, Osaka, Japan \\ Y. Ando \\ Department of Nephrology, Jichi Medical School, \\ Tochigi, Japan \\ S. Fujimoto \\ First Department of Medicine, Miyazaki University, \\ Miyazaki, Japan \\ T. Konta \\ First Department of Medicine, Yamagata University, \\ Yamagata, Japan \\ H. Yokoyama \\ Division of Nephrology, Kanazwa Medical University, \\ Ishikawa, Japan \\ H. Makino \\ Department of Nephrology, Diabetes and Rheumatology, \\ Okayama University Graduate School of Medicine, \\ Dentistry and Pharmaceutical Sciences, Okayama, Japan
}

\section{A. Hishida}

First Department of Medicine, Hamamatsu University School of Medicine, Shizuoka, Japan 
Table 3 Prevalence rates of CKD stages in Japanese adults (20 years or older), and estimated number of CKD cases per CKD stage based on the 2005 census

\begin{tabular}{llll}
\hline $\begin{array}{l}\text { GFR } \\
\left(\mathrm{ml} / \mathrm{min} / 1.73 \mathrm{~m}^{2}\right)\end{array}$ & Total & $\begin{array}{l}\text { Proteinuria } \\
(+)\end{array}$ & $\begin{array}{l}\text { Proteinuria } \\
(-)\end{array}$ \\
\hline $\begin{array}{l}\text { Prevalence rate }(\%) \\
\text { GFR } \geq 90\end{array}$ & 27.8 & 0.6 & 27.2 \\
$60-89$ & 61.6 & 1.7 & 60.0 \\
$30-59$ & 10.4 & 0.8 & 9.6 \\
$<30$ & 0.2 & 0.1 & 0.1 \\
Stage 3 & & & \\
$50-59$ & 7.6 & 0.4 & 7.2 \\
$40-49$ & 2.3 & 0.3 & 2.0 \\
$30-39$ & 0.6 & 0.1 & 0.4 \\
Estimated number of Japanese adults & 2005 & \\
GFR $\geq 90$ & 28639274 & 605313 & 28033961 \\
$60-89$ & 63576938 & 1708870 & 61868068 \\
$30-59$ & 10743236 & 823881 & 9919355 \\
$<30$ & 236569 & 125190 & 111379 \\
Stage 3 & & & \\
$50-59$ & 7809261 & 425146 & 7384116 \\
$40-49$ & 2363987 & 267158 & 2096828 \\
$30-39$ & 569988 & 131577 & 438411 \\
\hline
\end{tabular}

\section{S. Matsuo}

Department of Nephrology, Nagoya University Graduate School of Medicine, Nagoya, Japan 